\title{
Production, Detection and Study in the Infrared of Unstable Molecules and Radicals
}

\author{
Mireille Morillon-Chapey
}

Laboratoire de Physique Moléculaire et Applications, UPR 136, CNRS/Université Paris-Sud, Bât 350, 91405 ORSAY Cedex, France

\section{Introduction}

This subject is very wide. I shall present, here, a summary of our works related to radicals created in electric discharges. I shall review the methods and technical means used for studying these species in the infrared. This review will be accompanied with a detailed bibliography in which one will find the description of these methods and the most typical results obtained in our group in Orsay since 1986 .

\section{Scientific Aim of the Orsay Research Group}

It is mainly the spectroscopic characterization of very different media: interstellar matter, atmospheres, plasmas, flames etc...

\subsection{Our role is:}

To create the molecular species. This implies the set up of various sources (chemical reactions, electric discharges)

To obtain the spectral parameters i.e. to determine line position and line intensity constants.

To determine in the best case, the molecular dipole moment and potential functions.

\subsection{Our tools are:}

(a) A Fourier Transform Spectrometer (FTS) with the following characteristics:

- home built apparatus (Guelachvili 1978)

- recording mode: step by step

- maximum non-apodized resolution: $0.0027 \mathrm{~cm}^{-1}$

- large spectral domain: from the visible to $500 \mathrm{~cm}^{-1}$ 
- free spectral range up to $4000 \mathrm{~cm}^{-1}$

- precision of the wavenumber scale: $10^{-4}-10^{-5} \mathrm{~cm}^{-1}$

(b) A Tunable Diode Laser Spectrometer (TDLS) (Spectra Physics) used for real time monitoring of the plasma sources and studying line-broadening effects.

(c) A HP 9000-350 workstation to compute the spectra and to treat the data.

\subsection{The problems to solve are of different natures:}

- safety problems which need technical solutions

- intensity measurements by FTS which require understanding of complex instrumental effects

- production of molecules:

(a) by electric discharges with optimization of the sources and control of their stability by means of the TDLS. The impossibility of measuring the concentration of the molecules in the source leads to indirect determinations of transition moments.

(b) by chemical reactions: we must obtain samples of very high purity and keep and check this purity during the experiments. Pressure and temperature of the sample must be recorded during the experiments.

In spite of the fact that, at present, many of our works imply the use of chemical reactions in order to study molecules of atmospheric interest like $\mathrm{HNO}_{3}$, $\mathrm{ClNO}_{2}, \mathrm{ClONO}_{2} \ldots$ in this paper I will only present the techniques and results connected to the electric discharges.

\section{Electrical Sources of Radicals}

High concentrations of unstable molecules, in the laboratory, are generally difficult to obtain. This is mainly due to their short lifetime relative to their high chemical reactivity. Therefore we set up various experimental devices designed to efficiently produce unstable species. Figure 1 shows a simplified typical experimental configuration. The plasma reactor can be simultaneously observed by the diode-laser and Fourier-transform spectrometers.

The main sources that we use are:

(1) A radiofrequency reactor described by Chollet et al. (1986): It is a stainless tube, $3 \mathrm{~m}$ long and $22 \mathrm{~cm}$ in diameter, which contains a multiple reflection cell permitting optical paths of several tens of meters. The plasma is excited by a RF discharge. The first application of this device was the study of a Silane plasma emission spectrum in which we detected $\mathrm{SiH}$ : it was the first detection of this radical in the infrared (Chollet et al. 1986). The results of the $\mathrm{SiH}$ study are given by Betrencourt et al. (1986). These experiences showed that in this discharge the vibrational temperatures are higher than the rotational temperatures. The other molecules detected using a Silane plasma are: HNSi (Elhanine et al. 1991, 


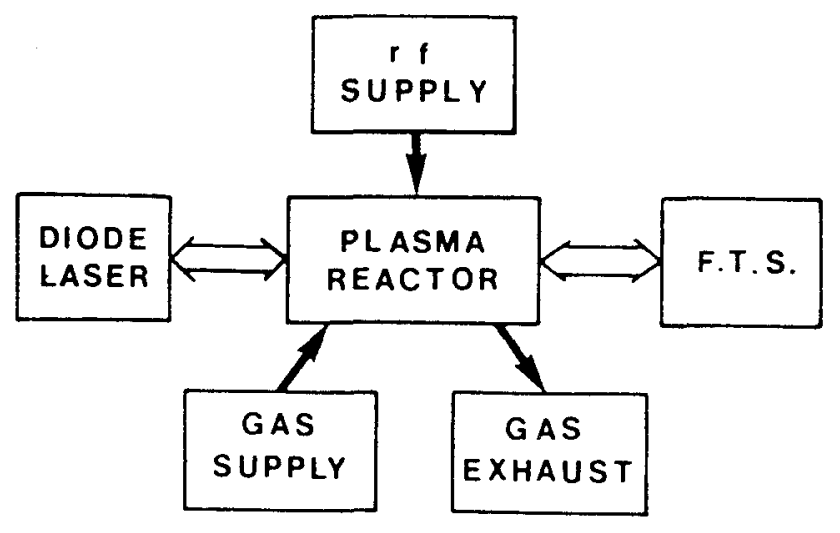

Fig. 1. The figure shows a simplified typical experimental configuration designed to efficiently produce and observe unstable species.

Elhanine et al. 1993) and SiN (Elhanine et al. 1992). Other types of plasma were achieved with the $\mathrm{RF}$ source: from a $\mathrm{H}_{2}+\mathrm{N}_{2}$ mixture we studied the $\mathrm{NH}$ radical (Boudjaadar et al. 1986) and from a $\mathrm{H}_{2} \mathrm{~S}$ plasma we studied $\mathrm{SH}$ and the negative ion $\mathrm{SH}^{-}$(Elhanine et al. 1988).

(2) A microwave source, well known for its advantages: (a) no electrodes and henceforth non impurities (b) wide pressure range (from $10^{-3}$ to 10 Torr), and (c) large density of generated species $\left(10^{12}-10^{13} / \mathrm{cm}^{3}\right)$. An important application was the observation of emission spectra of the $\mathrm{OH}$ radical from a $\mathrm{H}_{2} \mathrm{O}+$ $\mathrm{O}_{2}$ plasma. We obtained simultaneously pure rotational transitions in the $\mathrm{v}=0$ and $v=1$ states and the rovibrational fundamental band (Benidar 1991). The experiences showed a thermal equilibrium between vibration and rotation.

(3) A D.C. discharge in which we observe the emission of the positive column in the infrared. One characteristic of this often used discharge is that it allows to selectively excite the vibrations. For example, Benidar (1991) shows that for $\mathrm{OH}$ this discharge allowed the observation of the four first rovibrational bands with $\Delta v=1$. We hope to apply this possibility of highly excited vibrations to the $\mathrm{ClO}$ radical for an intensity study.

(4) A hollow-cathode discharge: an infrared emission spectrum of $\mathrm{N}_{2}^{+}$is described by Ferguson et al. (1992).

\section{Selective Detection of the Radicals}

A crucial problem linked to these sources is to discriminate the usually small signals due to the transient species from the large spectral background of the parent molecule or other stable species which are present at the same time. For this, a property of the species of interest, by which it differs from the precursors, must be chosen and varied in a well defined and periodic manner. In laser 
spectroscopy, modulation methods have proved to be very successful. However such methods have rarely been applied to high resolution FTS. In our group, new modulation schemes are being developed for the interferometer with the following specificity: the modulation of the output signal of the interferometer, essential to improve all the detections in FTS, is here, produced by the source in a selective manner and not by the interferometer itself. This selective manner depends on the nature of the species, as will be shown in a few examples.

(i) Paramagnetic species: We realized two types of modulation for these very usual species: (a) Zeeman-Modulation: constant and periodic magnetic fields are applied to the molecules in a cell. The effect of this source modulation on the spectrum is that only the lines which are field sensitive are detected as shown on NO (Elhanine et al. 1988) and (Guelachvili 1986). (b) Polarization modulation: based on the polarization property of the Zeeman transitions in a magnetic field. The principle and results are explained by Elhanine et al. (1989).

(ii) Short lived species: We showed that a selective detection by FTS is possible by modulating their production processes on a time scale similar to their lifetime. Thus the long-lived species will not be modulated and therefore not detected. An application to the $\mathrm{OH}$ radical detection in emission by concentration modulation is given by Benidar et al. (1991).

(iii) Molecular ions: The most powerful technique is the velocity modulation. It has been very successful in detecting many new ions with tunable infrared lasers, especially in the group of R.J. Saykally at Berkeley. We applied this technique to High-Resolution FTS with its well-known multiplex and wide spectral-range advantages. The principle is given by Martin \& Guelachvili (1990) with an application to $\mathrm{ArH}^{+}$, showing for the first time a velocity modulation in emission.

\section{Intensity Measurements}

It is well known that rovibrational intensities for radical species are particularly difficult to determine using experimental methods. However, the interaction between vibration and rotation (Herman-Wallis effect) can be exploited to determine the transition moment independently of any information on molecular abundance. The effect of this interaction results in a transfer of intensity in a band, from the $P$ branch to the $R$ one or vice-versa. The first spectacular evidence of this effect was seen on $\mathrm{SH}$ produced in the $\mathrm{SH}_{2}$ radio frequency plasma (Benidar et al. 1991). Then, thanks to this Herman-Wallis interaction, we studied the intensities of: NH (Chackerian et al. 1989), $\mathrm{ArH}^{+}$(Martin \& Guelachvili 1991) and OH (Benidar 1991).

Let us notice that, by this method we do not need any information upon the population distribution in the levels, i.e. this is valid even if the source is not in thermal equilibrium. This is shown on the $\mathrm{OH}$ emission spectra: as seen above we used spectra from two different sources (M.W and D.C. discharges). In the D.C. discharge the vibrational levels are higher and the rotational distribution does not follow a Boltzmann distribution as it does for the microwave 
discharge. Therefore, Benidar (1991) shows that these two quite different experiments lead to the same Herman-Wallis function and consequently to the same dipole moment function.

\section{References}

Benidar A., Farrenq R., Guelachvili G., Chackerian C., 1991, J. Mol. Spectrosc., 147, 383

Benidar A., 1991, These Universite Paris-Sud

Benidar A., Guelachvili G., Martin P.A., 1991, Chem. Phys. Letters, 177, 563

Betrencourt M., Boudjaadar D., Chollet P., Guelachvili G., Morillon-Chapey M., 1986, J. Chem. Phys., 84, 4121

Boudjaadar D., Brion J., Chollet P., Guelachvili G., Vervloet M., 1986, J. Mol. Spectrosc., 119, 352

Chackerian C., Guelachvili G., Lopez-Pineiro A., Tipping R.H., 1989, J. Chem. Phys., 90, 641

Chollet P., Guelachvili G., Morillon-Chapey M., Gressier P., Schmitt J.P.M., 1986, J. Opt. Soc. Am. B, 3, 687

Elhanine M., Farrenq R., Guelachvili G., 1991, J. Chem. Phys., 94, 2529

Elhanine M., Hanoune B., Guelachvili G., 1993, J. Chem. Phys., 99, 5

Elhanine M., Hanoune B., Guelachvili G., Amiot C., 1992, J. Phys., II 2, 931

Elhanine M., Farrenq R., Guelachvili G., Morillon-Chapey M., 1988, J. Mol. Spectrosc., 129,240

Elhanine M., Farrenq R., Guelachvili G., 1988, Mikrochim. Acta (Wien), II, 265

Elhanine M., Farrenq R., Guelachvili G., 1989, Appl. Opt., 28, 4024

Ferguson D.W., Rao K., Martin P.A., Guelachvili G., 1992, J. Mol. Spectrosc., 153, 599

Guelachvili G., 1978, Appl. Opt., 17, 1322

Guelachvili G., 1986, J. Opt. Soc. Am. B, 3, 1718

Martin P.A., Guelachvili G., 1990, Phys. Rev. Letters, 65, 2535

Martin P.A., Guelachvili G., 1991, Chem. Phys. Letters, 180, 344 